\title{
Technical Publication Transfer Test with McDonnell Aircraft Company: MIL-M-28001 (SGML) and MIL-D-28000 Class I (IGES)
}

\section{June 25, 1990}

Prepared by

Syscon Corporation

and Lawrence Livermore

National Laboratory

SYSCON contact

Jeff Howells

(619) 296-0085

LLNL Contact

Jill Farrell

(415) 423-6348

AFLC Contact

Mel Lammers

(513) 257-3085

Prepared for

Air Force Logistics Command AITI Project 


\section{Contents}

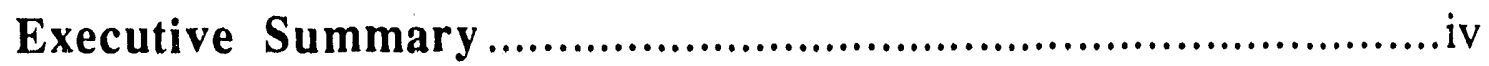

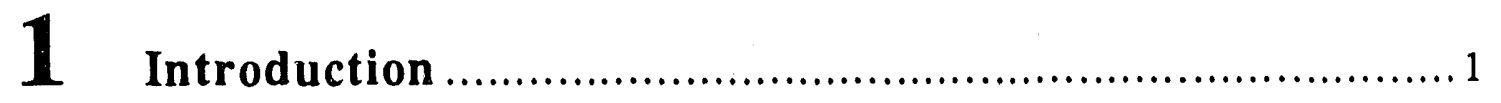

2 Test Description ............................................................

3 MIL-STD-1840A Analysis .........................................

4 MIL-M-28001 (SGML) Analysis .................................

5 MIL-D-28000 Class I (IGES) Analysis ...........................11

6 MIL-D-28003 (CGM) Analysis......................................17

7 Summary of Conclusions and Recommendations..............19

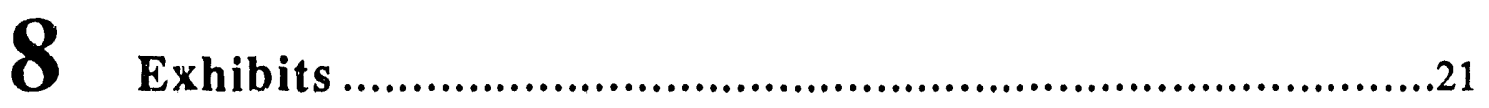




\section{Executive Summary}

The DoD Computer-aided Acquisition and Logistic Support (CALS) Test Network (CTN) is conducting tests of the military standard for the Automated Interchange of Technical Information (MIL-STD-1840A) and its companion suite of military specifications. The CTN is a DoD-sporsored confederation of voluntary participants from industry and government, managed jointly by a technical staff at Air Force Logistics Command (AFLC) and Lawrence Livermore National Laboratory (LLNL). The objective of CTN tests is to demonstrate and evaluate the interchange and functional use of digital technical information between industry and government using the CALS standards.

The subject of this report is a technical publication transfer to test MIL-STD-1840A, MIL-D-28000 Class I, MIL-M-28001, and MIL-D-28003. One focus of the test was the transfer of a moderately large volume of technical publication data. Data for the test originated at McDonnell Aircraft Company (MCAIR), located in St. Louis, Missouri. The test data was derived from the technical manual for the F-15E Aircraft Landing Gear System. The test was performed in accordance with part one of test plan CTN89-TM-06.

Non-conformance to MIL-STD-1840A included non-matching record values among document declaration records and corresponding data header records, a misspelled record header identifier, and an unexpected character in "filcnt:" record of the document declaration file. External packaging requirements were not analyzed.

There was some uncertainty on how the files should be segmented for Standard Generalized Markup Language (SGML) tagging purposes. If a document is to be delivered in separate files, the standard needs to include information on how to separate the files to be consistent with SGML requirements.

Only 10 Initial Graphics Exchange Specification (IGES) files were analyzed. Of these files, one had corrupted data. The rest of these files contained errors such as non-standard entities and misplaced graphics.

The Computer Graphics Metafile (CGM) data was not analyzed for this test since MCAIR had discovered that the files were incorrectly formatted. After the CTN had been notified of this problem, no further analysis was performed. However, MCAIR did correct the formatting problem and replacement metafiles were submitted to the CTN.

Recommendations that have resulted from this test include development of schemes for segmentation of text files to preserve SGML semantics, direct specification of CGM record and block specifications in the standard, elimination of "noise" characters in header records, development of variant DTDs to fit existing document families, specifications for external physical tape labels, and more information for product data. Finally, the standard should include more illustrations and examples. 


\section{Introduction}

The CALS Test Network (CTN) conducted a CTN Planned Test (CPT) of several CALS standards related to the transfer of technical publication data: MIL-STD-1840A (1840A), MIL-D-28000 Class I (28000), MIL-M-28001 (28001), and MIL-D-28003 (28003). The test was conducted with the McDonnell Aircraft Company (MCAIR) of St. Louis, Missouri in accordance with part one of CTN Test Plan CTN89-TM-06 and using material from an F-15E technical manual.

One focus of the test was the transfer of a moderately large volume of technical publication data. There were 9 text files, 74 Initial Graphics Exchange Specification (IGES) files, and 74 Computer Graphics Metafile (CGM) files in the exchange package. A noteworthy feature of the test was its early attempt to exchange CGM data.

Part one of CTN89-TM-06 called for technical publication data to be output from MCAIR's Xyvision system on 9-track magnetic tape. The data was to be in accordance with the 28001 subset of the Standard Generalized Mark-up Language (SGML) and the 28001 Document Type Definition (DTD) that conformed to MIL-M-38784B, the general style and format requirements for technical manuals. Technical illustrations in the manual were to be done in accordance with 28000 Class I (IGES) and 28003 (CGM).

Part two of the test called for the test tape to be read into another technical publication system where the text would be modified and the vector illustrations would all be converted to raster, in accordance with MIL-R-28002. This part of the test is not covered in this report.

\subsection{Purpose}

The purpose of this test is to obtain observations and to establish facts about digital data transfers that will support:

1. validation and improvements to $1840 \mathrm{~A}$;

2. conclusions regarding the feasibility of transferring moderately large volumes of technical publication data in conformance with $1840 \mathrm{~A}$;

3. evaluation of the usefulness to CTN test participants of software tools developed by the CTNO; and

4. analysis of the adequacy of the 28001 application of SGML. 


\section{An Automation Note:}

The title of $1840 \mathrm{~A}$ accurately portrays the intent: Automated Interchange of Technical Information. For CALS to be successful, the interchange must be automated to the optimum degree. The majority of the requirements of $1840 \mathrm{~A}$ must be met exactly in order that a given transfer proceed in the automated mode instead of the manually assisted mode. The reference here is to the requirements explicitly stated in $1840 \mathrm{~A}$, not those incorporated by reference to Military Specifications.

In this test and previous tests, deviations from these requirements were devised in order to proceed with the test and gain more insight into needed changes to the standard. 


\section{Test Description}

\subsection{Test Plan}

This test was conducted in accordance with part one of test plan CTN89-TM-06.

\subsection{Participants}

Evaluators:

1. SYSCON Corporation 3990 Sherman Street San Diego, CA 92110

2. Lawrence Livermore National Laboratory P.O. Box 808, L.542 Livermore, CA 94550

Test Data Originator:

3. McDonnell Aircraft Company Dept 500, Bldg 78, Lev 4, Post D1

St. Louis, MO 63166

\subsection{Test Data Description}

The MCAIR test data was derived from the technical manual for the F-15E Aircraft Landing Gear System, Technical Order 1F-15E-2-32GS-00-1.

The MCAIR test data consisted of three separate 1600 BPI tapes. The first tape contained the text data, the second the IGES data, and the third the CGM dat:. There were 9 text files, 74 IGES files, and 74 CGM files.

Note: MCAIR's CTN submittal was never intended to be a multi-volume tape submittal. It was submitted as three distinct tapes for ease of processing by the CTN, since the text would be processed by one site, the CGM illustrations by another, and the same illustrations in IGES format at yet another site. 


\subsection{Test Configuration}

SYSCON Test Platform:

Hardware:

DEC VAX 11/785 - VMS

Apple Macintosh II

SGML Parsers:

Datalogics INPAR (4.1); VAX

SoftQuad MARK - IT (1.0); Mac II

Software Exoterica Checkmark (1.1b13); Mac II

1840A Tape

Evaluation Tools: CTN TAPEVAL(0.9); VAX

\section{LLNL Test Platform:}

Hardware:

MicroVAX II - VMS

Sun $3 / 60$ - UNIX

IGES File Verification: Glatz Associates IGES Model Testing System (4.3); VAX

IGES File Viewing: Rosetta Technologies, Inc. PrePARE and PreVIEW (2.0 Beta); SUN

MCAIR Test Platform:

Hardware: $\quad$ Xyvision, Inc. FPS 95

Xyview III Terminal

IBM 4381

Evans and Sutherland Graphics Terminal

SGML Parser: $\quad$ Sobemap Version 2.0

IGES File Generation: McDonnell Doulgas CADD/CATI System

1840A Tape: Xyvision, Inc. ANSI Tape Utility

\subsection{Standards Tested:}

MIL-STD-1840A (1840A)

MIL-D-28000 Amendment 1 (28000) Class I

MIL-M-28001 (28001)

MIL-D-28003 (28003)

\subsection{Special Preparations}

No special preparations were required for this test. 


\section{MIL-STD-1840A Analysis}

\subsection{Test Results}

The MCAIR data was not in conformance with the requirements specified by 1840 A. A problem included non-standard external physical tape labels. However, all of the text and illustration (IGES and CGM) files were correct in record format and size. All block sizes for each data type were also correct.

The document declaration files contained an incorrectly spelled record 9 , "dtetrn: ". Also, all records were terminated with a colon. This is not inconsistent with $1840 \mathrm{~A}$ in the document declaration header records, except for the "filcnt:" record (record 11). In this instance, the colon character generated an error in the CTN Tapeval Program.

Another error centered around the fact that the "filcnt:" record specified illustration files without any associated text files to reference them, since the illustrations and text were on separate tape sets. According to current interpretation of $1840 \mathrm{~A}$, each technical publication illustration file must have an associated text file that references it.

All text file header records were correct, but both the IGES and CGM files had header records that were not equivalent to their corresponding records in the document declaration file. This stemmed from the colon character that was in different positions in the fixed length records of the CGM and IGES files than in the variable length records of the data declaration file.

\subsubsection{Conformance to Packaging Standards}

The MCAIR test data was not evaluated for conformance to packaging standards. Future test reports will be subject to evaluation of packaging.

\subsubsection{External Tape Labeling}

The MCAIR test data was delivered on three separate magnetic tapes. Each tape contained two labels. One specified the name of the originating organization and the associated address as well as the destination organization and address.

The second label contained a label title of "CALS TEST NETWORK," "standard 9-track 1600 BPI ANSI TAPE LBL," "D001," "D001Q001 D001Q074." Although this label contained most of the pertinent information, it did not identify what each piece of individual information was. For instance, D001 was on a line by itself. This appeared to be the tape label but was not specified as such. Similarly, the filenames should be identified. 


\subsubsection{Analysis of the Tapes}

The external tape labels were not standard on the three tapes that were received. However, the structure of the IGES data met the requirements of 1840A. All IGES files were correct in record size and format ( 80 byte, fixed); all block sizes were valid on the tape (2000 bytes). Similarly, the text file structure looked good. All text files were correct in record size and format ( 256 byte variable size records); all block sizes were valid on the tape (2048 bytes).

Evaluation of the log files indicated that the CGM files were fixed length records of 80 characters with a block length of 800 bytes. MIL.STD-1840A does not specify record characteristics for CGM data so the validity of the CGM data in this respect can not be verified until the standard is modified.

The aggregate of all the data was more than one tape could hold. In such a case, $1840 \mathrm{~A}$ requires the data to be delivered as a multi-volume transfer. Instead of a multi-volume tape set, the data was delivered as three separate tapes. One tape contained all CGM files, another all of the IGES, and the last one contained all text files. At the beginning of each tape was a document declaration file with the filename "D001." MIL-STD-1840A requires that the document declaration files reside at the beginning of the tape set and precede all associated data files. This was not done since the data was sent as separate tapes.

\subsubsection{Analysis of the Document Declaration Files}

Each of the three tapes contained a document declaration file and in each case identical errors were observed with the same records of each document declaration file. The first error involved an incorrect spelling of record 9, "dtetrn:". The second error encountered was an illegal colon as the last character in record 11 ("filcnt:"). The colon was found as the last character in each of the document declaration header records. While the colon is not illegal in these records, it is not needed and generated an error in the CTN analysis tools.

The document declaration files on the IGES and CGM tapes also generated the following message using the CTN tools. "**** ERROR - pending resolution of $1840 \mathrm{~A}$ standard issues. Current interpretation requires an associated text file for graphic data files." This error message relates to the requirement that every graphic must have an associated text file that references it if it is technical publication data. This requirement was not satisfied by the provided test data since the text files and illustration files were delivered on separate tapes and not contained in one logical document.

\subsubsection{Analysis of 1840A Text Header Records}

All text header records were present and contained legal values. It was noted, however, that the last character in each record was a colon. 


\subsubsection{Analysis of 1840A IGES Header Records}

Two consistent errors were identified after analysis of the IGES header records. These errors were detected in each of the 74 IGES header files. The first error was that the "srcdocid:" record (record 1) of the IGES file did not match the corresponding "srcdocid:" record of the document declaration file. Further analysis indicated that this error resulted from a colon at character position 80 in the IGES file which was not in the same position in the document declaration file.

The second error was similar to the previous error except that it occurred in the "dstdocid:" records. Again, this error was the result of a colon that appeared in different character locations in the IGES and corresponding document declaration file. The colon character also appeared in the remaining records.

\subsubsection{Analysis of 1840A CGM Header Records}

CGM header records contained the same two problems that existed in the IGES files. Again, these problems occurred as a result of a colon in the "srcdocid:" and "dstdocid:" records of the CGM file that did not appear in the same position in the corresponding records of the document declaration file.

Unnecessary colons also appeared at the end of each of the remaining records though they did not generate any errors.

\subsection{Conclusions and Recommendations}

Several recommendations, relating to the transmission envelope, have resulted from this test. The first is that a few areas of $1840 \mathrm{~A}$ need to be more clearly defined, such as section 4.1.1.2.5. This section should state that the requirement for each illustration file to be referenced by an accompanying text file applies only to technical publication data and not engineering data.

Second, 1840 A should also specifically state that only printable characters are permitted in the header records. Non-printable "noise" characters, such as line feeds and null characters, should not be allowed. The colon character should also not be allowed. Such characters serve no useful purpose, and in cases of the "filcnt:" and "dtetrn:" records, generate illegal character parsing errors when using the CTN evaluation tools. In this case, the colons were actually added by MCAIR due to a misinterpretation of $1840 \mathrm{~A}$. The standard should more clearly state that just the record identifier string, not the entire header record, should be concluded by a colon.

Third, header record values of fields common with the document declaration file such as the "srcdocid:" and "dstdocid:" must be exact matches, on a character by character basis. The standard should emphasize this fact. 
Fourth, 1840A needs to contain clear documentation, with examples, specifying the record formats and block sizes of CGM data since this is not directly stated.

Finally, in order to avoid the confusion indicated in 3.1.2, section 5.3 of $1840 \mathrm{~A}$ should have examples of external tape labels and packing slips.

In addition, to avoid problems with misspellings and stray characters in $1840 \mathrm{~A}$ document declaration files and data header records, the CTN recommends that automated tools for writing 1840A tapes be used where ever possible. For this test, MCAIR hand generated the declaration and header records. To avoid future errors, the company now has software in place to generate entire $1840 \mathrm{~A}$ tapes automatically. 


\section{$4 \quad$ MIL-M-28001 (SGML) Analysis}

\subsection{Test Results}

The MCAIR text files were delivered as individual files (option C). The standard supports this method allowing each of the individual document elements to be located in an individual file. The 1840A standard, however, does not define guidelines for segmenting the files with respect to SGML tagging requirements. Many SGML parsers will only accept a complete document for input. This necessitates a reconstruction of the document from the individual document component. A problem arises in this situation since files that were constructed to parse as stand alone entities may not parse correctly as a collection of concatenated files. If "dummy" tags were inserted in the stand alone files to permit a successful parse, then they should be explicitly defined as such to enable their removal if a complete document reconstruction is desired.

After the MCAIR files were reconstructed, several tagging errors were detected. Each of the errors was repairable. Error severity had a broad range. Less severe errors included missing quotes with the tag attributes. More serious errors involved document content problems such as the a $<$ para0 $>$ following a <abbrsect $>$.

The text files were contained in nine separate files. These files were concatenated to serve as valid parser input. In addition, each of the text files contained a Document Type Definition (DTD) which is allowed. To enable further evaluation, the DTDs were removed.

MIL-M-28001 was used as the reference DTD. Amendment 1 of this standard was not used since it was not available when this test was originally constructed. The Software Exoteric Checkmark package, hosted on the Apple Macintosh, was the parser used for SGML evaluation.

The following problems were discovered. It should be noted as a general fact that all problems cited pertain only to these text files when parsed with the conforming DTD and as the data files were concatenated. These tags may be valid when parsed with the DTD that was submitted with the cata. The following content model problems were observed in the instance.

1. A < contents $>$ should not follow a $<$ lep $>$ in this instance.

2. A $<$ para $>$ is not allowed in a $<$ para0 $>$.

3. All <para0 $>$ and $<$ subpara $1>$, <subpara $2>$, <subpara3>, etc., must be followed by a $<$ title $>$.

4. <para0> can not follow an abbreviation section tag, <abbrsect>. 
As a recommendation, the standard should be updated to inclucle a scheme on the segmentation of text files so that the reconstructed files are consistent with respect to 28001 or allow the individual files to te parsed separately.

\subsubsection{DTD Compiiance}

No agreement was made regarding the use of a DTD other than the one found in the appendix of 28001 . The MCAIR test data included a DTD as pari of each of the text files. The MCAIR DTDs were not identical to the 28001 standard DTD.

The format of this document cannot be made to fit the DTD found in 28001 . MCAIR chose to modify the "conforming" DTD found in 28001 and code the document in accordance with the modified DTD, a practical choice for a production environment. There are thousands of documents in current use tha, follow the same general formatting conventions as this test document. The structural differences between the generation of documents represented by this test sample and the current 28001 DTDs can only be accommodated for transfer and databasing purposes by constructing a variant DTD that fits the history represented by these documents.

\subsubsection{Output Specification Compliance}

No Output Specification data was provided with the test data.

\subsection{Conclusioris and Recommendations}

Analysis of the text files has shown that scme aspects of the preparation of the data was not consistent with the $1840 \mathrm{~A}$ standard.

It is recommended that $1840 \mathrm{~A}$ be revised to include information on the segmentation of text files containing SGML tags. Specific SGML information should be defined that specifies the exact procedures for transmitting documents that have been divided into smaller files. These procedures should also consider the fact that the segregated files of a document may have to be reconstructed to enable correct input format for many SGML parsers and for imaging.

It is also recommended that a suite of variant DTDs be developed to fit existing types of documents that do not conform to the standard DTD found in 28001 . 


\section{MIL-D-28000 Class I (IGES) Analysis}

\subsection{Test Results}

The 74 illustrations were drafted three-dimensionally at MCAIR on a CADD/CATI System and then pre-processed with the "Class I" option into IGES files. This option translated all entities into two dimensions and attempted to map all entities to allowable Class I entities.

Ten of the 74 illustrations were evaluated in depth. These ten drawings were selected for their differing illustration types, i.e. electrical schematic, tabular text, isometric appearance, etc., and for their likelihood of containing frequently incorrectly processed IGES entities. Illustrations 1 , $8,21,24,29,31,37,39,41$, and 70 were the ones chosen, and plots of 1 , 31 , and 41 are displayed in the Exhibit section of this report. Their names correspond to their orde ing within the technical publication.

The analysis involved checking conformance to the standards and plotting the stored graphics. The conformance of each file to 28000 Class I and to IGES was verified with the IGES Model Testing System produced by Glatz Associates. The files were then graphically viewed and plotted with the PreVIEW Package by Rosetta Technologies, Inc.

The testing revealed interesting results regarding the 28000 specification, MCAIR's pre-processor, and MCAIR's procedures for preparing the data. Table I summarizes the results. The table indicates the existence of usable data and identifies specific problems for each illustration in three categories: conformance to 28000 Class I, conformance to the IGES specification, and correctness of the graphics. Table I shows that all analyzed files were "usable" accept one. "Usable" means that the data was readable by a post-processor and that it correctly embodied most of the graphics. The "unusable" file (illustration 39) contained a stray character in the beginning of the IGES data that created an incorrect IGES format and rendered the file useless. Speculation is that the computer operator entered the stray character by accident while hand-editing the $1840 \mathrm{~A}$ header information into the IGES file. This error demonstrates the need for automated $1840 \mathrm{~A}$ tape writing tools to reduce user-induced errors.

Regarding conformance to 28000 Class I, the table reveals that only one file conformed completely, and that the others were in non-conformance due to problems in the pre-processor and/or within 28000 itself. Specifically, MCAIR's pre-processor placed illegal 28000 Class I entities, "Copious Data - Data Points" (106 Form 3), into three of the IGES files. MCAIR is aware of the problem and has already addressed it in a new software version. 
Table I - MIL-D-28000 Class I (IGES) Test Results in Tabular Form

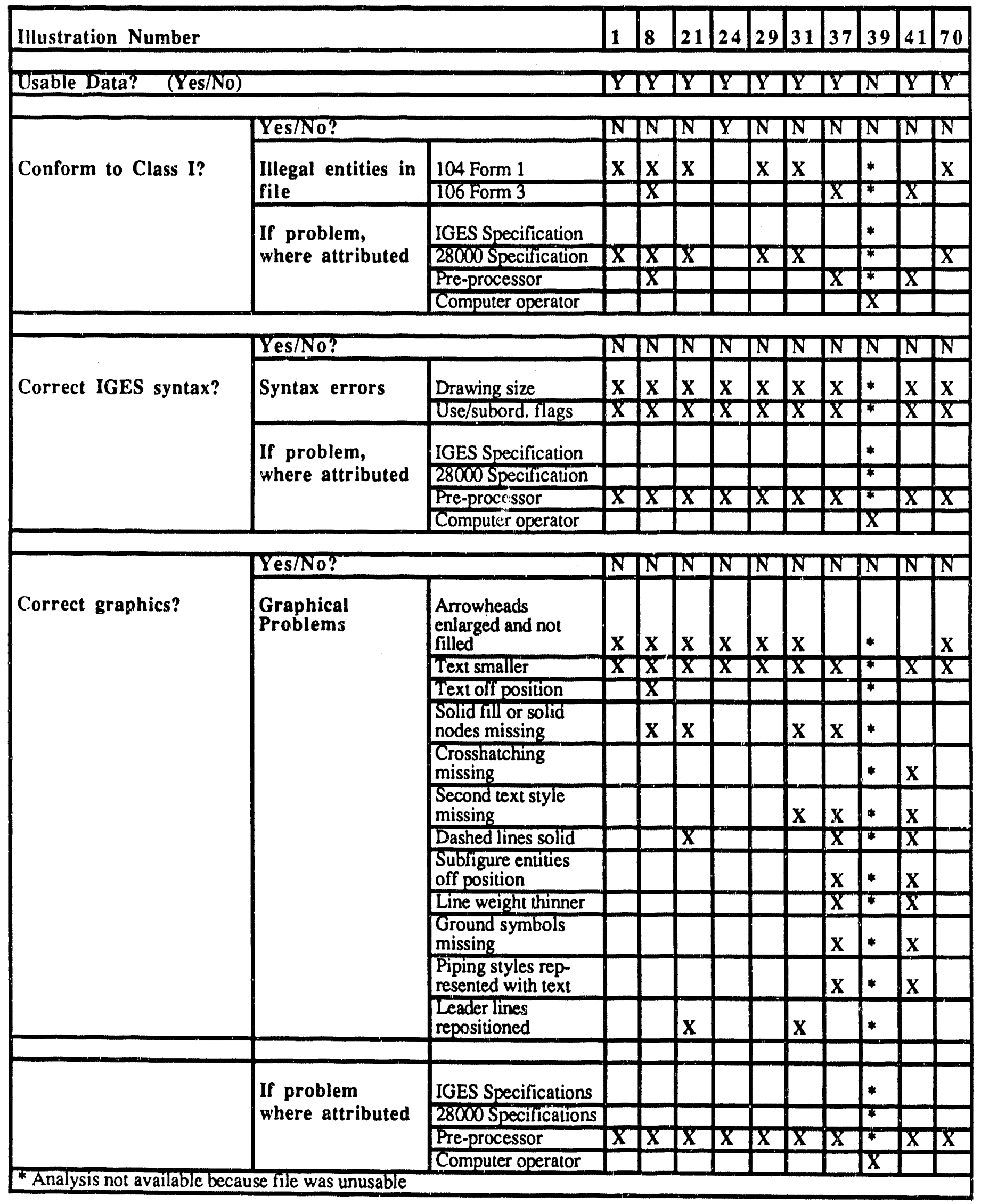


Furthermore, the table shows that "Conic Arc - Ellipse" entities (104 Form 1) appear in MCAIR's IGES files even though they are not allowable Class I entities (Class I only allows the "Conic Arc - General" entity). After discussing this issue with the chairperson of the technical group that created the Class I subset, it is believed that Class I was supposed to allow all forms of the "Conic Arc" entity and that this restriction is a mistake in 28000. The CTN technical staff will recommend a correction to this oversight.

Regarding conformance to IGES syntax, Table I shows that all files were found to have at least one problem, however the errors would not likely affect the data transfer process. The problems included incorrect drawing sizes within the "Drawing Size Property" entities, and various incorrectly set "Use" and "Subordinate Entity" flags. In all cases the problems were. attributed to the illustration system's pre-processor.

Lastly, regarding correctness of the graphics within the files, Table I indicates none of the 10 illustrations translated perfectly. Exemplary plots of the illustrations before and after pre-processing are displayed in the Exhibit section of this report. A comparison of these plots revealed that some of the IGES files contained nearly perfect representations of the graphics that the original illustrations contained. They displayed only minor graphical inconsistencies in text size, arrowhead size, and arrowhead fill. Other files contained other levels of disparities, from missing crosshatching and dashed lines to misplaced subfigures. All problems with graphics were attributed to the IGES pre-processor, although many may have been corrected in MCAIR's new software release.

\subsection{Conclusions and Recommendations}

The CTNO technical staff is pleased to see that computer-aided design and illustration vendors are implementing 28000-subset-compliant IGES processors. Although in this case the implementation was not perfect, the usability of the data was quite good. Besides identifying problems in the pre-processor, this test also highlighted some needed procedural changes as well as 28000 modifications. As a result of this testing, it is recommended that:

1. McDonnell Aircraft Company continue to improve its implementation of the 28000 Class I processor.

2. The CTNO technical staff and others continue to create software tools that help auromate the writing of $1840 \mathrm{~A}$ tapes, especially where large amounts of $1840 \mathrm{~A}$ data are involved.

3. The appropriate CALS Committee change the entity name from "Conic Arc - General" to "Conic Arc" on Table I - The Technical Publication Illustration Subset, in 28000, Amendment 1. 


\section{MIL-D-28003 (CGM) Analysis}

\subsection{Test Results}

The original MCAIR CGM files were incorrectly formatted as indicated by MCAIR during the analysis phase of this test. Once this was known, no further analysis was applied.

The problems identified were corrected, and replacement metafiles were submitted to the CTN of the ten graphics being tested. It should be noted that the CTN is currently using some of the CGMs from this test group as examples of valid MIL-D-28003 CGMs. 


\section{Summary of Conclusions and Recommendations}

Non-conformance to $1840 \mathrm{~A}$ included non-matching record values among document declaration records and corresponding data header records, a misspelled record header identifier, and an illegal character in the "filcnt:" record of the document declaration file. External packaging requirements were not analyzed.

The text files contained several tagging errors ranging in severity when parsed with the conforming DTD instead of the DTD submitted. There was some uncertainty on how the files were segmented for tagging purposes. If a document is to be delivered in separate files, the standard needs to include information on how to separate the files to be consistent with SGML requirements.

No agreement was made regarding the use of a DTD other than the one found in the appendix of 28001. The MCAIR test data included a DTD as part of each of the text files. The MCAIR DTDs were not identical to the 28001 standard DTD.

The format of this document cannot be made to fit the DTD found in 28001 . MCAIR chose to modify the "conforming" DTD found in 28001 and code the document in accordance with the modified DTD, a practical choice for a production environment. There are thousands of documents in current use that foll iw the same general formatting conventions as this test document. The structural differences between the generation of documents representud by this test sample and the current 28001 DTDs can only be accommodated for transfer and databasing purposes by constructing a variant DTD that fits the history represented by these documents.

MCAIR's IGES files were drafted and then pre-processed on a McDonnell Douglas CADD/CATI System. Ten of the 74 illustrations were evaluated in depth. The evaluation of these illustrations involved analyzing the files for conformance to 28000 Class I, viewing the graphics stored in the IGES files, identifying problems, and recommending possible solutions.

In general, the illustrations transferred well. Of the 10 illustrations: 3 were nearly perfect in graphical appearance, syntax, and conformance; 6 contained both non-28000 Class I entities and a few missing or misplaced graphics; and 1 had corrupted data such that the file was unusable. One of the problerns was attributed to a misprint in 28000 and another to user errors, but most of the problems were attributed to the IGES pre-processor of MCAIR's illustration system.

After analysis of the $1840 \mathrm{~A}$ test data several conclusions can be drawn. First, it is essential that the standard be followeo exactly. Many errors that occurred in the header records were the result of a colon character that appeared in different positions. In the case of large amounts of data, the data should be delivered as a multi-volume tape set as specified by the standard. 
This test has also indicated several areas of $1840 \mathrm{~A}$ for which information is lacking, or needs to be modified as follows.

1. Block sizes and record format specifications for CGM data.

2. In the case of common header record values, such as the "srcdocid:" of the document declaration file and associated text, IGES, raster, etc. files, it should be explicitly defined that these records should be identical on a character by character basis (i.e. positional).

The CTN technical staff is pleased to see that computer-aided design and illustration vendors are implementing 28000-subset-compliant IGES processors. Although in this case the implementation was not perfect, the usability of the data was quite good. Besides identifying problems in the pre-processor, this test also highlighted some needed procedural changes as well as 28000 modifications. As a result of this testing, it is recommended that:

1. McDonnell Aircraft Company continue to improve its implementation of the 28000 Class I processor.

2. The CTN technical staff and others continue to create software tools that help automate the writing of $1840 \mathrm{~A}$ tapes, especially where large amounts of $1840 \mathrm{~A}$ data are involved.

3. The appropriate CALS Committee change the entity name from "Conic Arc - General" to "Conic Arc" on Table I - The Technical Publication Illustation Subset, in MIL-D-29000, Amendment 1.

The original MCAIR CGM fil's were incorrectly formatted as indicated by MCAIR during the analysis "yase of this test. Once this was known, no further analysis was applied.

In summary, this test has shown that it is crucial to follow $1840 \mathrm{~A}$ exactly. In view of the fact that computer automated methods will be used to verify this data, it must be in strict conformance. Manual and subjective data analysis will not be feasible. Another major result of this test is that it has identified areas of the standard where information is lacking or needs to be more concise. It is recommended that the standard be updated in these areas to permit the construction and transmittal of consistent and accurate data. 


\section{Exhibits}

The following exhibits are plots of three illustrations studied during this test. These illustrations were selected because they show some of the typical graphical inconsistencies between the original and translated versions of the illustrations after translation through MCAIR's IGES pre-processor. Illustrations 1, 31, and 41 are displayed in "original" and "as transferred" form.

Exhibit 1: $\quad$ MCAIR's Illustration 1 as originally drawn and plotted.

Exhibit 2: $\quad$ MCAIR's Illustration 1 after IGES translation. Note the smaller text and misshaped arrowheads.

Exhibit 3: $\quad$ MCAIR's Illustration 31 as originally drawn and plotted.

Exhibit 4: $\quad$ MCAIR's Illustration 31 after IGES translation. Note the missing solid fill and misplaced arrow.

Exhibit 5: $\quad$ MCAIR's Illustration 41 as originally drawn and plotted. (Size reduced from a fold-out to a single page.)

Exhibit 6: $\quad$ MCAIR's Illustration 41 after IGES translation. Note the missing crosshatching, the misplaced subfigures, and piping styles that are represented with text. (Size reduced from a fold-out to a single page.) 


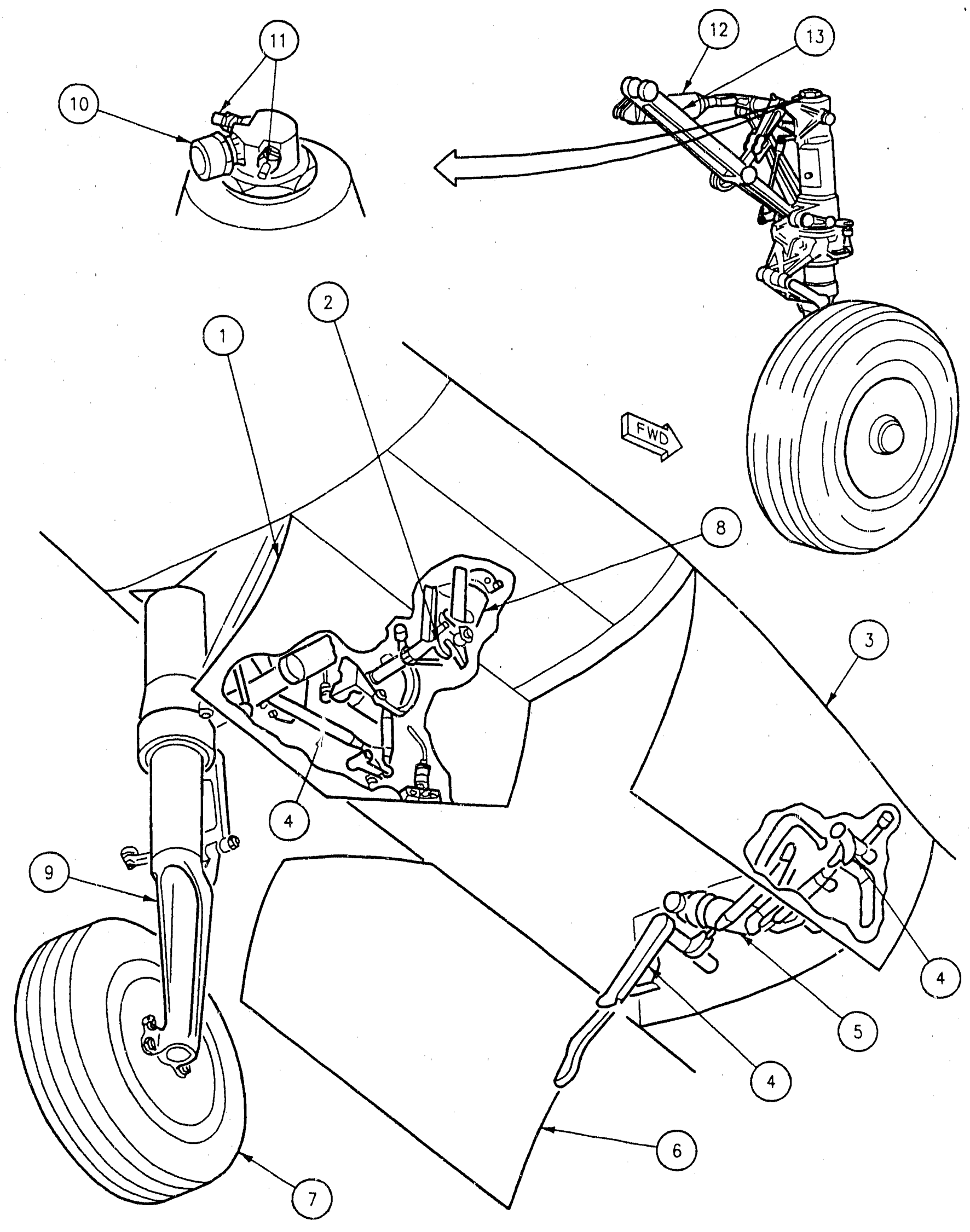

$15 E-32 G 5-00-(17-1)-S C A N$

Exhibit 1: $\quad$ MCAIR's Illustration 1 as originally drawn and plotted 


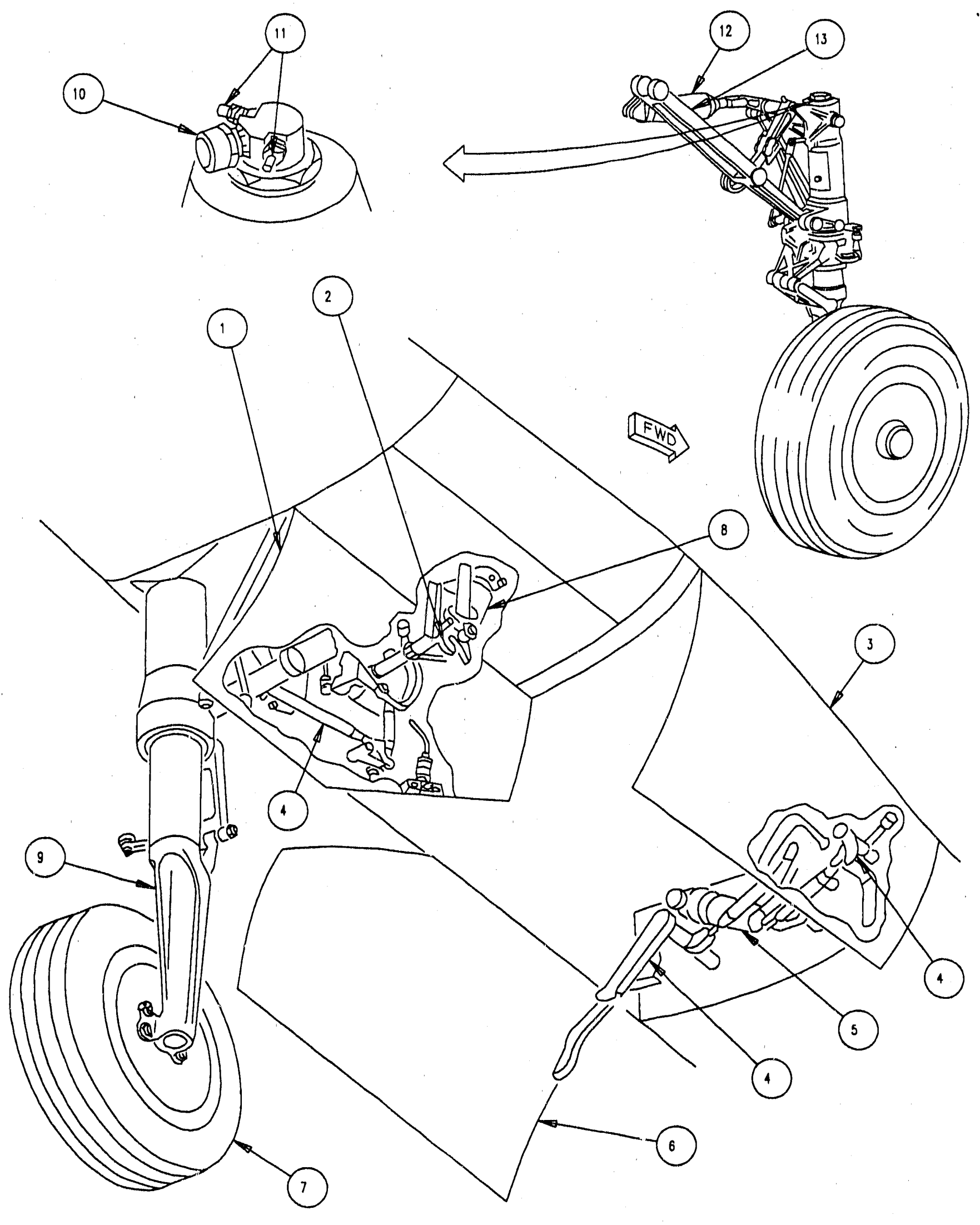

$16-2 x-60-617-12-x+24$

Exhibit 2: $\quad$ MCAIR's Illustration 1 after IGES translation.

Note the smaller text and misshaped arrowheads. 


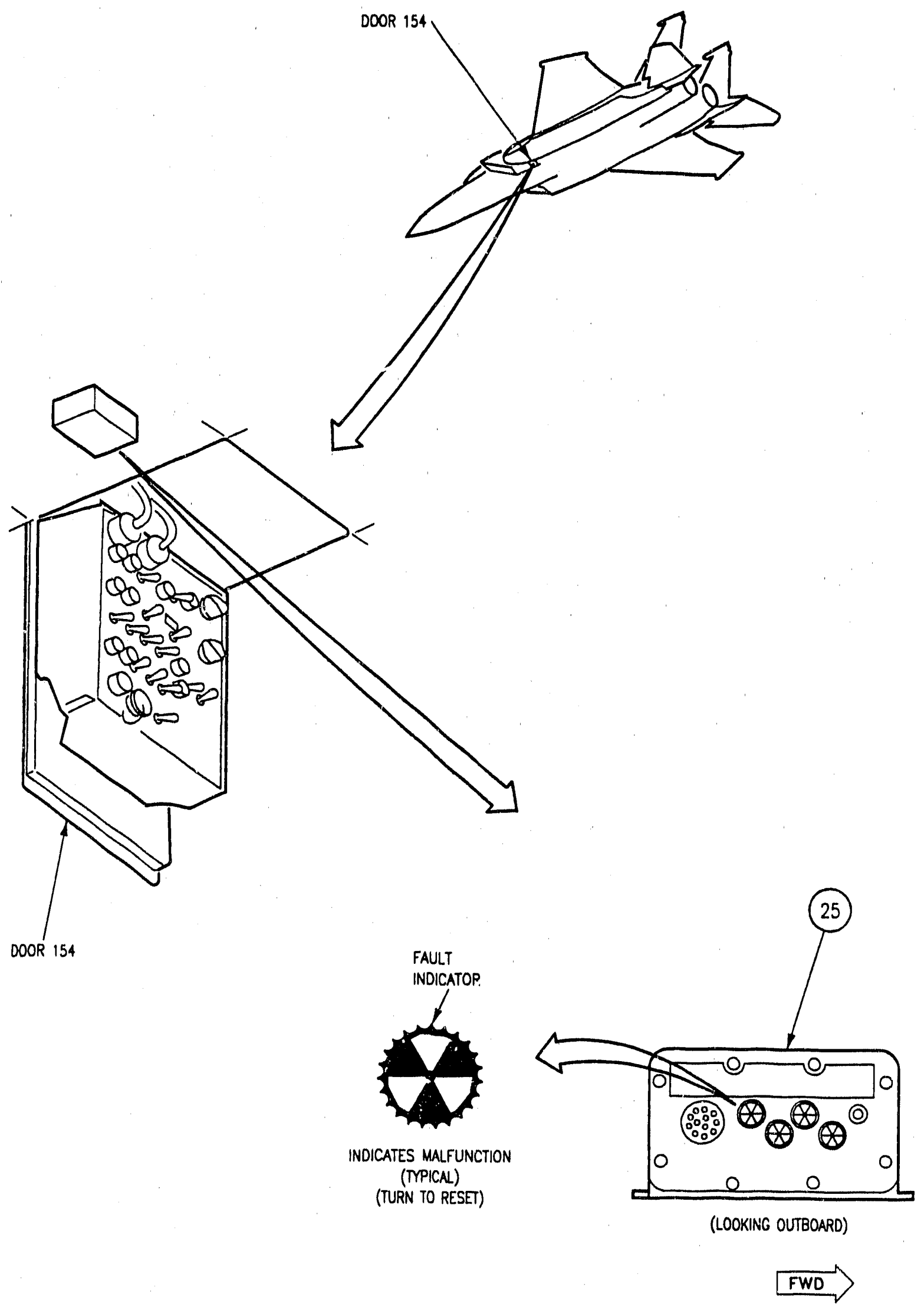

$15 E-3200-00-(9-8)-\operatorname{cAT}$ 


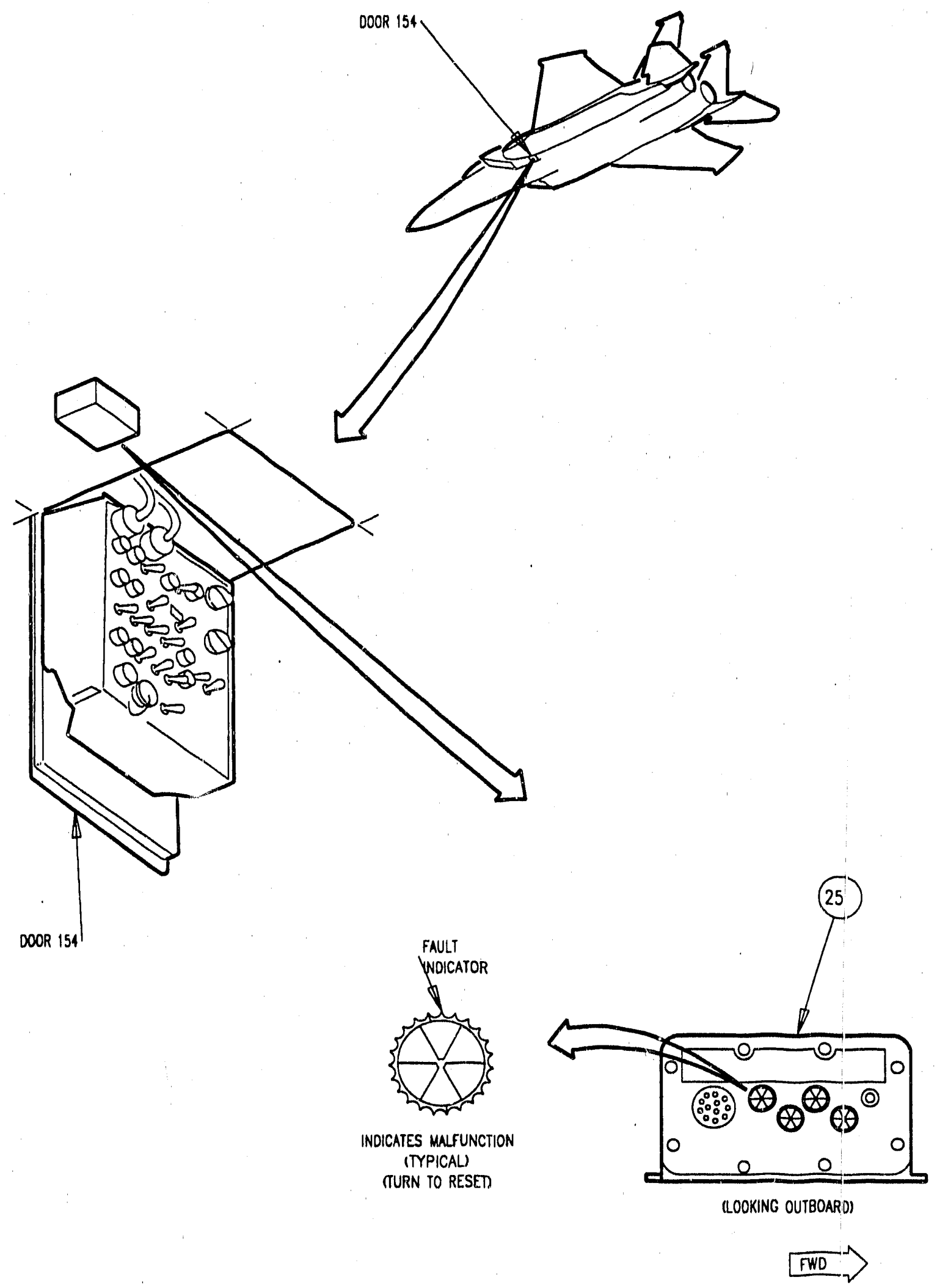

$15[-3205-00-(0-8)-\operatorname{can}$

Exhibit 4: $\quad$ MCAIR's Illustration 31 after IGES translation.

Note the missing solid fill and misplaced arrow. 


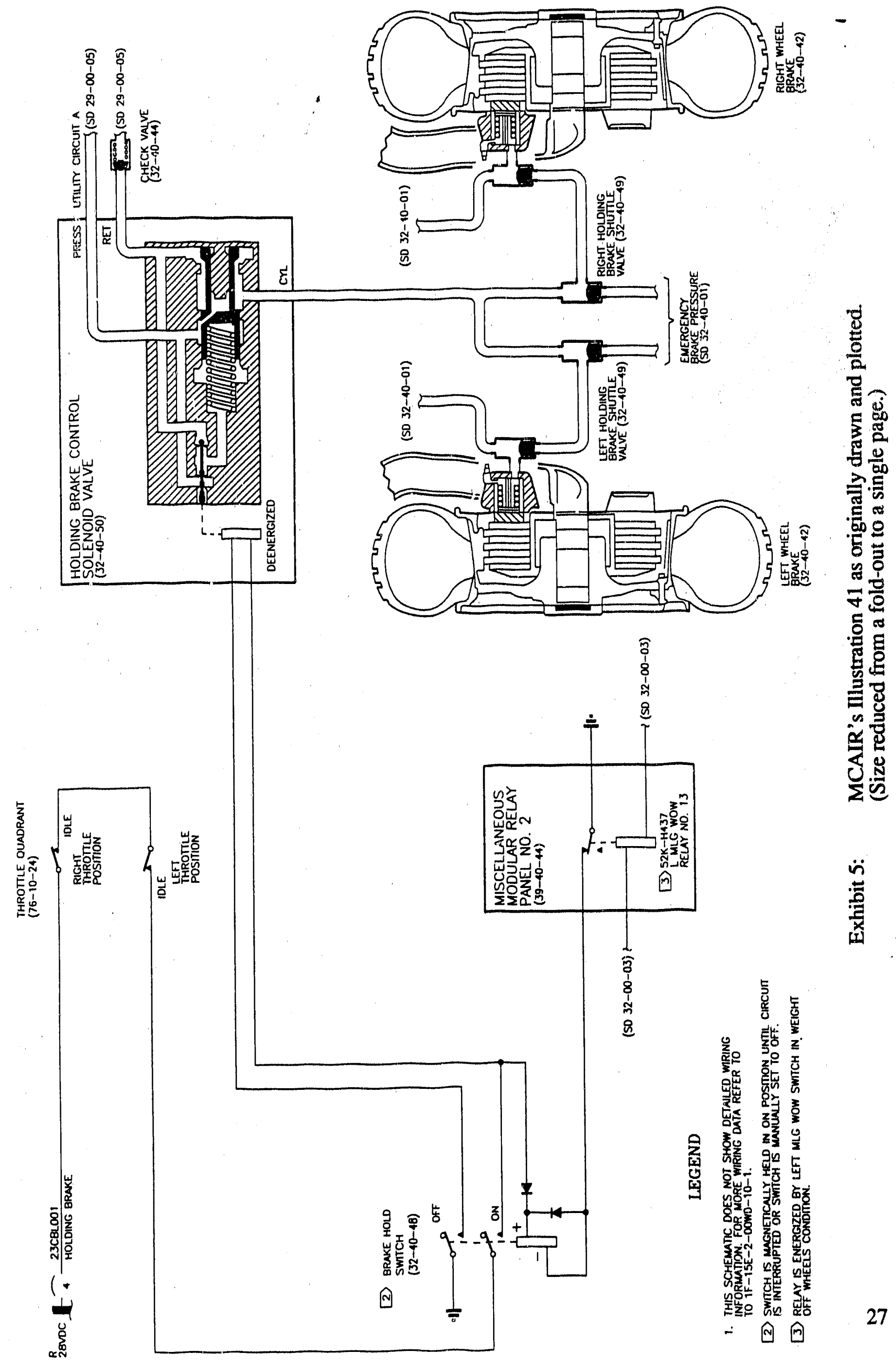




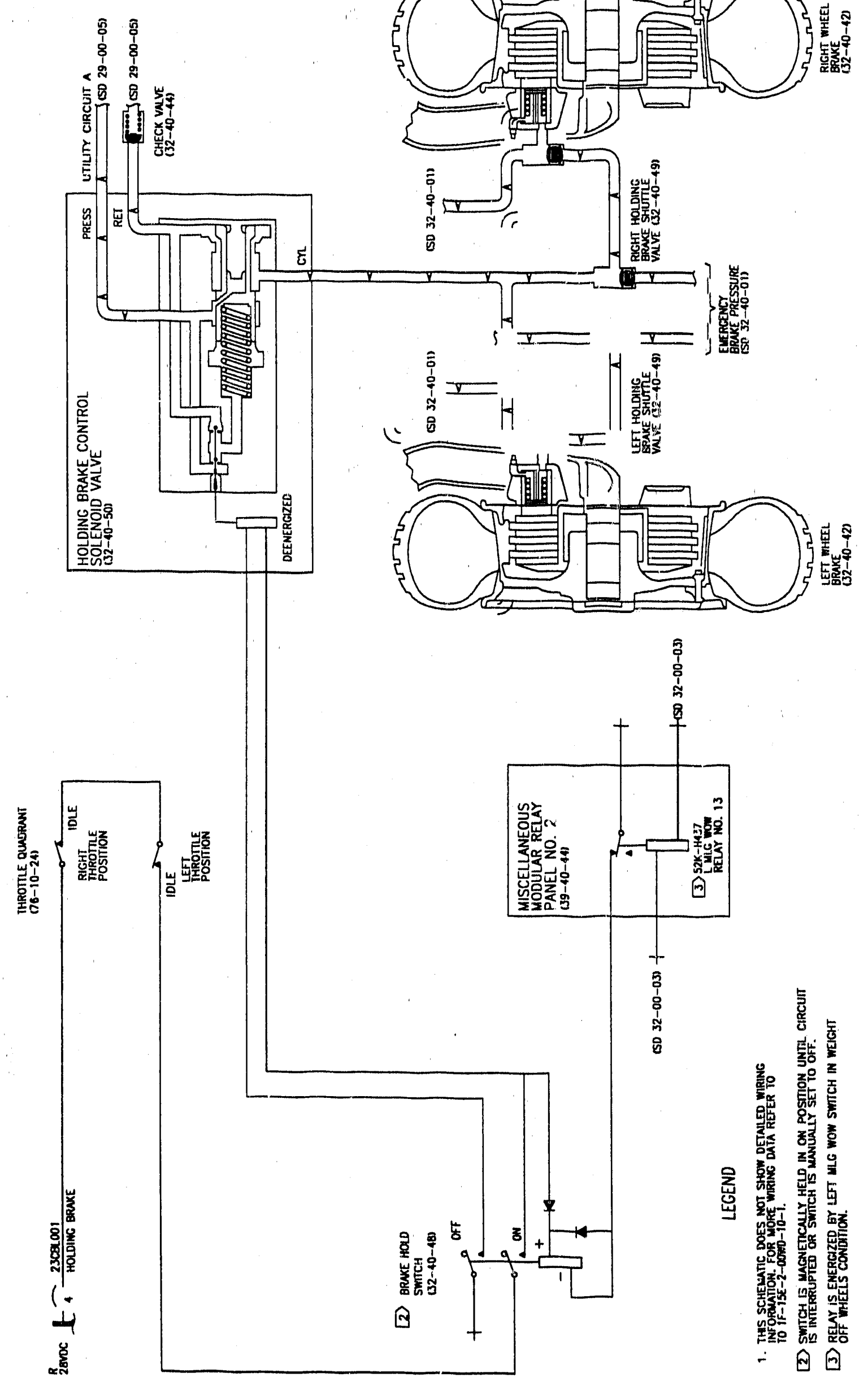

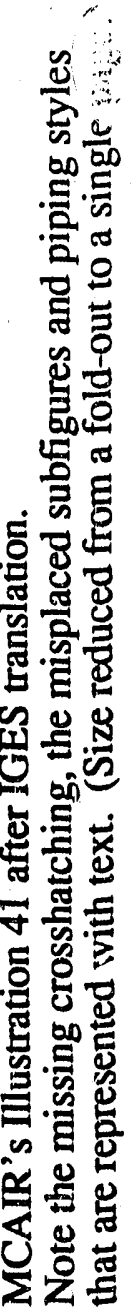



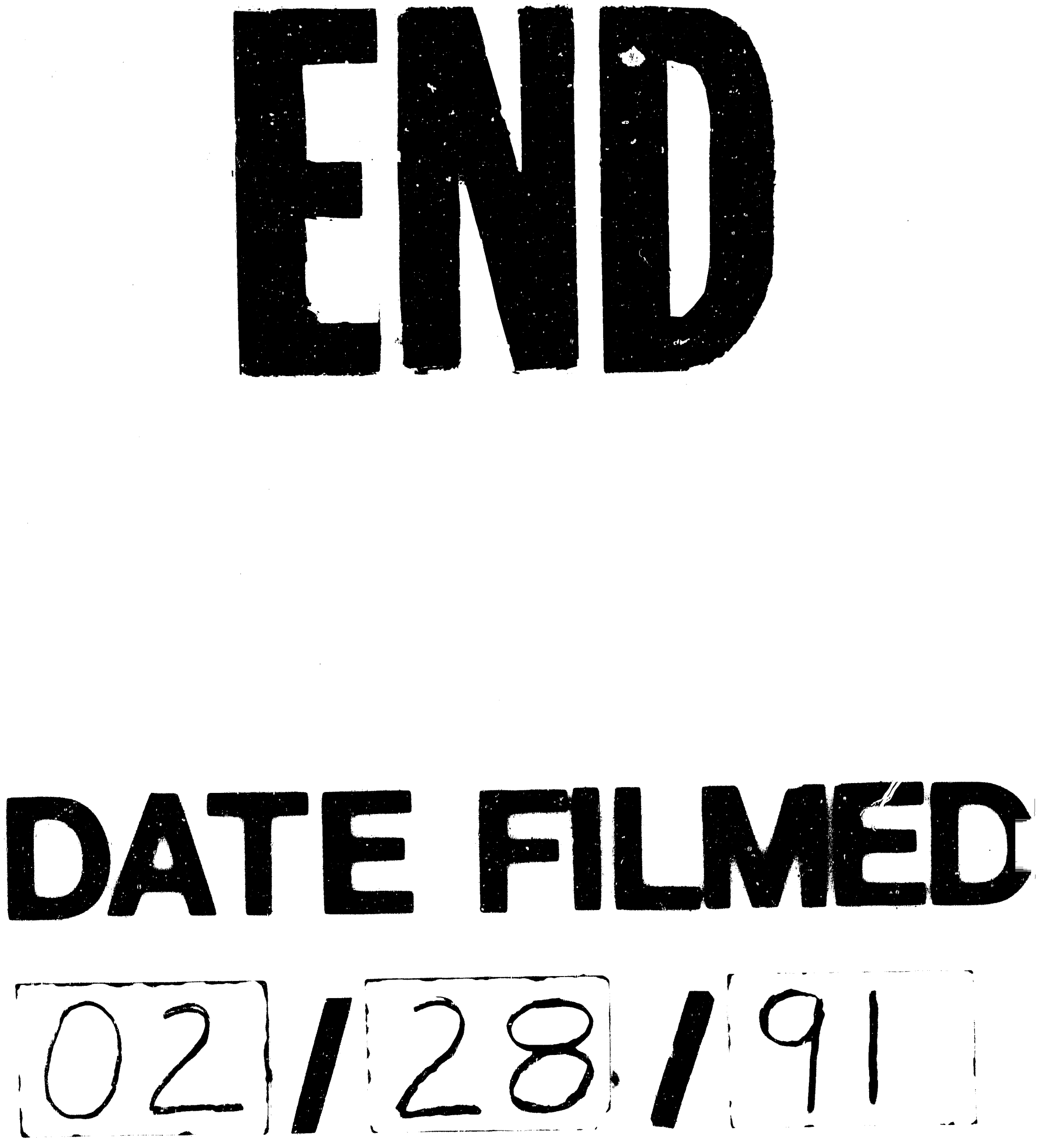


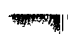

